\title{
Feasibility and safety study of 22-gauge endoscopic ultrasound (EUS) needles for portal vein sampling in a swine model
}

\section{다)(우우}

\author{
Authors \\ Kenneth Park ${ }^{1}$, Daniel Lew ${ }^{1}$, Christopher Chapman², Ashley Wachsman ${ }^{1}$, Matthew Bloom ${ }^{3}$, Liiana Bancila ${ }^{1}$, Rachel \\ Perry ${ }^{1}$, Qiang Wang ${ }^{1}$, Laith Jamil ${ }^{4}$, Stephen Pandol' ${ }^{1}$, Simon Lo ${ }^{1}$
}

Institutions

1 Cedars-Sinai Medical Center, Division of Digestive Diseases, Los Angeles, California

2 University of Chicago Medical Center, Center for Endoscopic Research and Therapeutics, Chicago, Illinois, United States

3 Cedars-Sinai Medical Center - Surgery, Los Angeles, California, United States

4 William Beaumont Hospital - Royal Oak, Gastroenterology and Hepatology, Royal Oak, Michigan, United States

submitted 29.5.2020

accepted after revision 20.8.2020

Bibliography

Endoscopy International Open 2020; 08: E1717-E1724

DOI 10.1055/a-1264-7206

ISSN 2364-3722

(C) 2020. The Author(s).

This is an open access article published by Thieme under the terms of the Creative Commons Attribution-NonDerivative-NonCommercial License, permitting copying and reproduction so long as the original work is given appropriate credit. Contents may not be used for commecial purposes, or adapted, remixed, transformed or built upon. (https://creativecommons.org/licenses/by-nc-nd/4.0/)

Corresponding author

Kenneth H. Park, Cedars-Sinai Medical Center -

Gastroenterology, 8700 Beverly Blvd, Suite 7700, Los Angeles, California 90048-0750, United States

Fax: +1-310-423-1826

kenneth.park@cshs.org

\section{ABSTRACT}

Background and study aims Endoscopic ultrasound (EUS) has been used for portal vein sampling in patients with pancreaticobiliary cancers for enumerating circulating tumor cells but is not yet a standard procedure. Further evaluation is needed to refine the methodology. Therefore, we evaluated the feasibility and safety of 19-gauge (19G) versus a 22-gauge (22G) EUS fine-needle aspiration needles for portal vein sampling in a swine model.

Methods Celiotomy was performed on two farm pigs. Portal vein sampling occurred transhepatically. We compared $19 \mathrm{G}$ and $22 \mathrm{G}$ needles coated interiorly with saline, heparin or ethylenediaminetetraacetic acid (EDTA). Small- (10 mL) and large- $(25 \mathrm{~mL})$ volume blood collections were evaluated. Two different collection methods were tested: directto-vial and suction syringe. A bleeding risk trial for salinecoated $19 \mathrm{G}$ and $22 \mathrm{G}$ needles was performed by puncturing the portal vein 20 times. Persistent bleeding after 3 minutes was considered significant.

Results All small-volume collection trials were successful except for 22G saline-coated needles with direct-to-vial method. All large-volume collection trials were successful when using suction syringe; direct-to-vial method for both $19 \mathrm{G}$ and $22 \mathrm{G}$ needles were unsuccessful. Collection times were shorter for $19 \mathrm{G}$ vs. $22 \mathrm{G}$ needles for both small and large-volume collections $(P<0.05)$. Collection times for saline-coated $22 \mathrm{G}$ needles were longer compared to heparin/ EDTA-coated $(P<0.05)$. Bleeding occurred in $10 \%$ punctures with $19 \mathrm{G}$ needles compared to $0 \%$ with $22 \mathrm{G}$ needles. Conclusion The results of this animal study demonstrate the feasibility and the safety of using $22 \mathrm{G}$ needles for portal vein sampling and can form the basis for a pilot study in patients.

\section{Introduction}

Pancreatic ductal adenocarcinoma (PDAC) is one of the deadliest forms of human cancers [1]. Endoscopic ultrasound (EUS) has been widely used for tumor staging and biopsy of pancreatic lesions [2]. More recently, EUS has emerged as a minimally invasive approach to access the portal system for measuring por- tal pressure in patients with chronic liver disease $[3,4]$ and collecting portal blood samples for analysis of circulating tumor cells (CTCs) in patients with pancreaticobiliary diseases [5]. Recent studies show that liquid biopsy, including characterization of CTCs and the circulating tumor DNA (ctDNA), present theoretical advantages over conventional tissue biopsy and may provide useful information on disease progression, metastatic 
risk, and clinical outcomes [6-13]. For most gastrointestinal cancers, CTC levels in the portal venous blood are higher than those in peripheral blood, due to the route of circulation and the filtering effects of the liver and the peripheral capillaries $[5,14-16]$. Analysis of portal venous CTC or ctDNA has potential value in guiding management of patients with gastrointestinal cancers. However, current literature is limited in regard to the methodology of collecting portal blood samples with EUS. Only three studies have evaluated EUS-guided portal vein sampling, and each study used different needle sizes including 19gauge EUS-fine-needle aspiration (FNA) needles, 20-gauge to 21-gauge EUS-FNA needles, and 22-gauge EUS-FNA needles [5, 17-19].

Given the increasing application of EUS-guided portal vein access and sampling, it is important to further evaluate and refine the methodology. The objectives of this study were to evaluate the feasibility and safety of using a 19-gauge (19G) versus a 22-gauge (22G) EUS-FNA needle for portal vein sampling.

\section{Methods}

\section{Animal model}

The animal study was carried out by following a protocol approved by the Cedars-Sinai Institutional Animal Care and Use Committee (IACUC protocol \#008428). A swine model was selected because it was considered the best alternative to human subjects for the proposed work, based on similarities in anatomy and portal vein pressure [20].

Two farm pigs (one male $39.5 \mathrm{~kg}$, one female $30 \mathrm{~kg}$ ) were used. Anesthesia was performed by the Cedars-Sinai Comparative Medicine staff under the IACUC approved core protocol (\#008428), followed by celiotomy performed by the study staff surgeon. After 12 hours of fasting, the study animals underwent general anesthesia with cardiopulmonary monitoring. With the pigs placed in the supine position, access to the abdominal cavity was gained through a standard $20-\mathrm{cm}$ incision ranging from approximately $7 \mathrm{~cm}$ above the umbilicus to approximately $9 \mathrm{~cm}$ below the umbilicus. The abdominal wall was retracted with a Bookwalter system. An intraoperative transhepatic ultrasound was performed by an experienced radiologist to identify the left intrahepatic portal vein, and Doppler was used to verify the flow signal. Once the left intrahepatic portal vein was identified, either a $19 G$ or a $22 \mathrm{G}$ EUS-FNA needle (Cook Medical, EchoTip Ultra, Bloomington, Indiana, United States) was advanced, by hand, into the vein for aspiration (>Fig.1 and PFig.2). A "U-loop" mimicking the expected bending of the EUS needle shaft within an echoendoscope was created during the aspiration. The pigs were monitored throughout the procedure (pulse, blood pressure, oxygenation), and were euthanized under anesthesia immediately following the procedures.

\section{Intraoperative procedures}

An experienced interventional endoscopist performed all intraoperative procedures on the two pigs, which included portal vein sampling and bleeding trials.

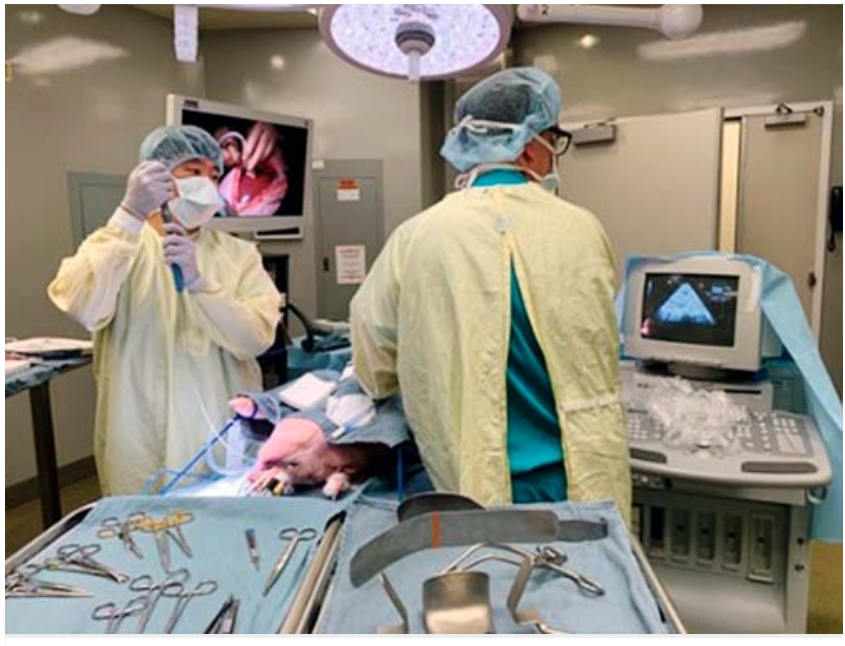

Fig. 1 The ultrasound probe is being used to identify the left intrahepatic vein and the EUS-FNA needle is being prepared for portal vein aspiration.

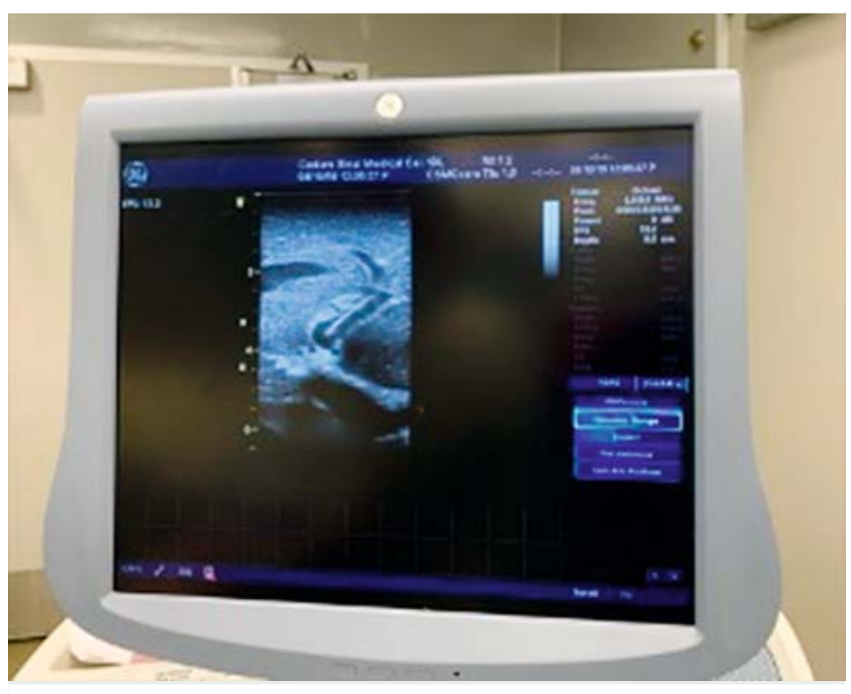

Fig. 2 Ultrasound showing the EUS-FNA needle successfully inside the left intrahepatic portal vein.

\section{Portal vein sampling}

Portal vein sampling was carried out on two pigs. For the first pig, we evaluated $19 \mathrm{G}$ and $22 \mathrm{G}$ needles coated with saline vs. heparin or ethylenediaminetetraacetic acid (EDTA), two anticoagulants commonly used to prevent clotting. Needles smaller than $22 \mathrm{G}$ were not tested based on significantly increased collection time required on the preliminary ex vivo tests with $25 \mathrm{G}$ needles and thus increased likelihood of clotting due to a decreased flow rate. Furthermore, the amount of blood required for our study was not feasible using $25 \mathrm{G}$ needles. Pressure build-up with a small needle channel might also lead to disruption of cellular structures or shearing. Prior studies evaluating CTCs have used sodium heparin (16.5 units/mL of blood) and sodium EDTA as an anticoagulant in collection vials [5,21]. Therefore, we tested the efficacy of using heparin and EDTA to prevent clotting in the EUS needle compared to no treatment. 

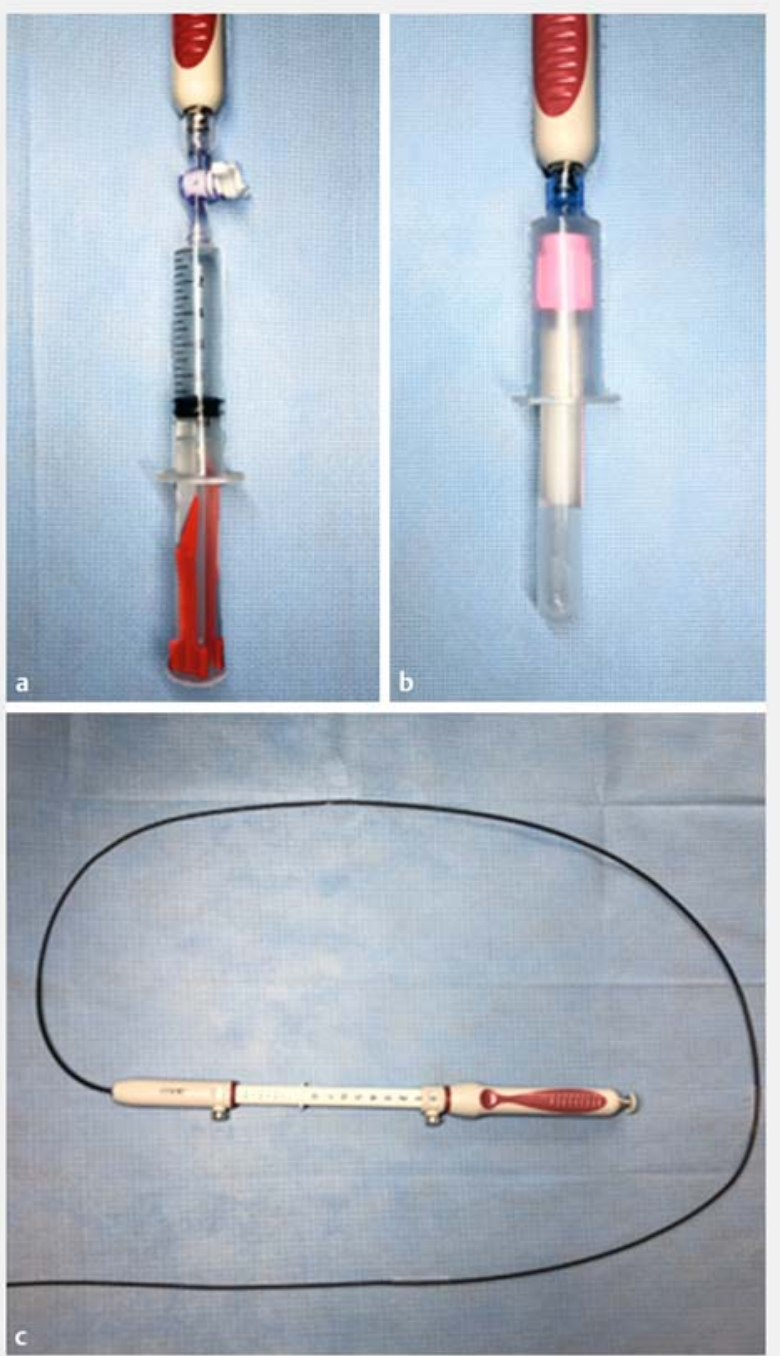

- Fig. 3 Instruments used during the study for portal vein sampling. The suction syringe collection method is shown in Panel A. The direct-to-vial method is shown in Panel B with the Vacutainer Luer Adapter attached directly to the EUS-FNA needle. Panel C shows the EUS-FNA needle.

This was performed by initially flushing the EUS needle with either heparin $(1000 \mathrm{U} / \mathrm{mL})$ or EDTA $(1.8 \mathrm{mg} / \mathrm{mL})$ after removing the stylet. The stylet was then re-introduced back into the needle, which removed the majority of the fluid from the needle shaft except for approximately $100 \mathrm{uL}$, or $100 \mathrm{U}$ heparin sodium and 1.8 ug of EDTA, for both $19 \mathrm{G}$ and $22 \mathrm{G}$ needles. We conducted both small-volume and large-volume collection trials. Two different collection methods were tested-a direct-to-vial (attaching a Vacutainer Luer Adapter directly to the EUS needle) and a traditional $10 \mathrm{~mL}$ suction syringe transfer to vacutainer ( $\triangleright$ Fig. 3 and $\triangleright$ Fig.4). Collection time, clot formation, and bleeding complications were recorded. Complete blood collection was defined as successful collection of 5-mL blood samples per tube, and collection time was defined as time needed to collect $5 \mathrm{~mL}$ of blood per vial.

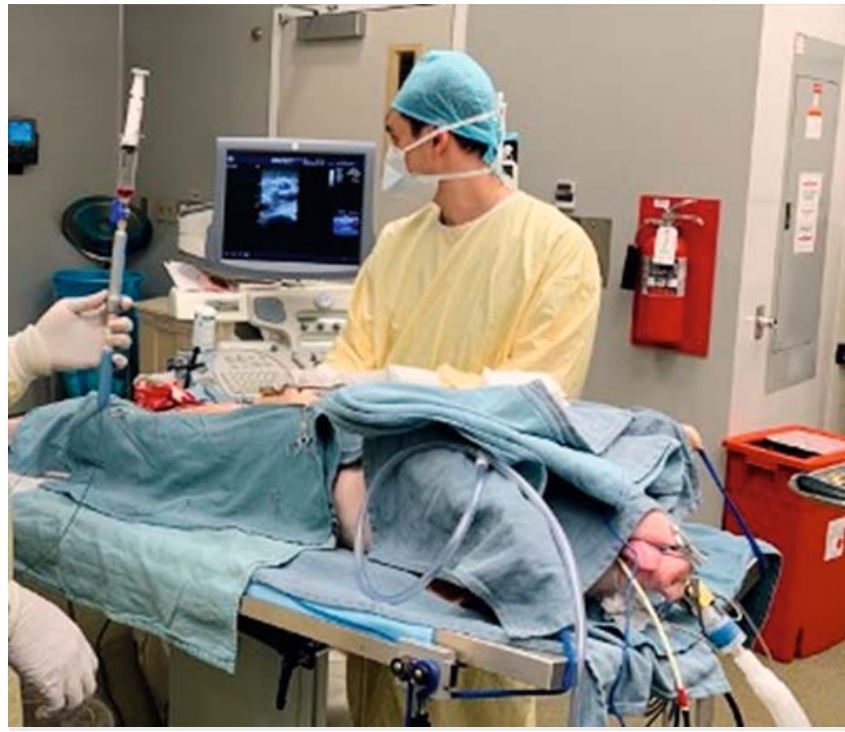

-Fig. 4 Example of portal vein sampling using the suction syringe collection method. The suction syringe is attached to the EUS-FNA needle, and blood is collected via negative pressure.

\section{Small-volume collection trials}

Small-volume collection trials consisted of collecting $10 \mathrm{~mL}$ of blood $(2 \times 5-\mathrm{mL}$ tubes) per needle. We conducted three trials: first trial with saline-coated needles, second trial with heparincoated needles, and the third trial with EDTA-coated needles. Within each trial, $1 \times 19 \mathrm{G}$ needle was compared to $1 \times 22 \mathrm{G}$ needle using direct-to-vial method, and $1 \times 19 \mathrm{G}$ needle was compared to $1 \times 22 \mathrm{G}$ needle using the suction syringe method.

\section{Large-volume collection trials}

The second pig was used to compare the feasibility and safety of collecting a large volume of blood using only heparin-coated needles. Large-volume collection trials consisted of collecting $25 \mathrm{~mL}$ of blood $(5 \times 5 \mathrm{~mL}$ tubes) per needle. We conducted three trials. Within each trial, $1 \times 19 \mathrm{G}$ needle was compared to $1 \times 22 \mathrm{G}$ needle using direct-to-vial method, and $1 \times 19 \mathrm{G}$ needle was compared to $1 \times 22 \mathrm{G}$ needle using the suction syringe method. A brand new EUS needle was used for each different trial.

\section{Blood cell enumeration}

The initial set of portal venous blood samples were collected and analyzed for complete blood count to assess for evidence of any significant hemolysis and any significant shearing forces encountered during the collection.

\section{Bleeding trials}

The bleeding trials were conducted on one pig and consisted of needle punctures performed 20 times for each needle gauge. One $19 \mathrm{G}$ and one $22 \mathrm{G}$ saline-coated needle was used for all 20 needle punctures. After using ultrasound to confirm the correct needle placement in the intrahepatic portal vein, the needle was removed, and the puncture site was assessed for bleeding under direct visualization for 3 minutes without external tam- 
ponade. Any ongoing bleeding after 3 minutes that required external tamponade was recorded.

\section{Statistical analysis}

Data are presented as mean \pm standard deviation. The $t$-test was employed for comparison of quantitative data. $P<0.05$ was considered statistically significant. One-way analysis of variance with post-hoc Tukey's tests was used for multiple comparisons between needle treatments (no treatment $v$ s heparin vs EDTA). $P<0.05$ was considered statistically significant.

\section{Results}

\section{Small-volume collection trials}

When using the suction syringe method, the collection times for saline-coated $22 \mathrm{G}$ needles were longer compared to needles coated with either heparin or EDTA (mean $171 \mathrm{sec}$ vs. 137 sec or $143 \mathrm{sec} ; P<0.05$ ). The collection times for saline-coated $19 \mathrm{G}$ needles were not significantly affected by heparin or EDTA treatment (mean $13 \mathrm{sec}$ vs. $11 \mathrm{sec}$ or $10 \mathrm{sec}$; $P>0.05$ ) ( Fig. 5).

With the direct-to-vial method, blood collection using saline-coated $22 \mathrm{G}$ needles was not completed due to clotting issues, which was completely resolved by needles coated with heparin (mean $162 \mathrm{sec}$ ) or EDTA (mean $171.5 \mathrm{sec}$ ) (> Fig. 6). In comparison, blood collection was successful using salinecoated 19G needles (mean $26.14 \mathrm{sec}$ ), and the blood collection time was not significantly altered by the treatment of the needles with either heparin (mean $25.5 \mathrm{sec}$ ) or EDTA (mean $24 \mathrm{sec}$ ) $(P>0.05)$. With both the 19G and $22 \mathrm{G}$ needles, there were no significant differences observed between heparin and EDTA groups ( $P>0.05)$.

When comparing direct-to-vial method to suction syringe method using the heparin/EDTA-coated 19G/22 G needles, there were no significant differences in mean blood collection times between the two methods ( $>$ Table 1 ).

Overall, the use of $19 \mathrm{G}$ needles led to shorter collection times compared to $22 \mathrm{G}$ needles regardless of anticoagulant treatment or collection method ( $\triangleright$ Fig. 5 and $\triangleright$ Fig. 6 ; $P<0.05$ ).

For the 19G needles coated with either heparin or EDTA, complications included mild bleeding at the time of puncture, but resolved within 3 minutes. In the first trial with the EDTAcoated $22 \mathrm{G}$ needles, clotting occurred initially but resolved after flushing the needle again with EDTA. No complications were observed with the heparin-coated $22 \mathrm{G}$ needles.

\section{Large-volume collection trials}

Given that the heparin-coated $22 \mathrm{G}$ needles did not have any complications and successful completion was demonstrated with smaller volumes, only heparin-coated needles were used to compare the efficacy and safety of the different needle gauges in this large-volume $(25 \mathrm{~mL})$ collection trial.

Both $19 \mathrm{G}$ and 22G heparin-coated needles had complete blood collection using the suction syringe method. The mean collection time for the $22 \mathrm{G}$ needle was longer compared to that of the $19 \mathrm{G}$ needle (mean $187 \mathrm{sec}$ vs $19 \mathrm{sec} ; P<0.05$ ) ( $\vee$ Fig.7). There were no issues with regard to clot formation or bleeding.

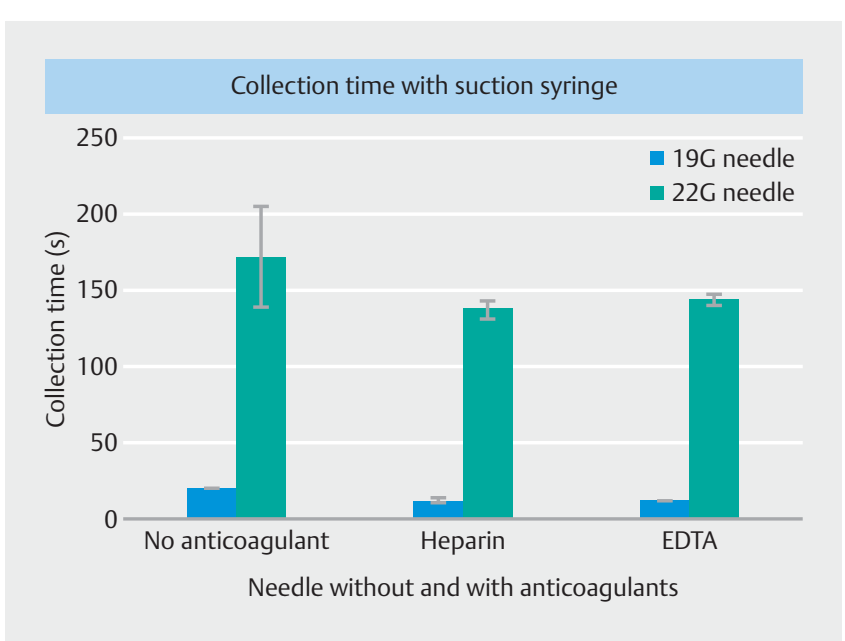

- Fig. 5 Mean small-volume $(10 \mathrm{~mL})$ blood collection times with suction syringe using 19G/22 G needles with or without anticoagulants. $22 \mathrm{G}$ needles coated with heparin or EDTA had significantly shorter blood collection times compared to saline-coated needles $(P<0.05)$.

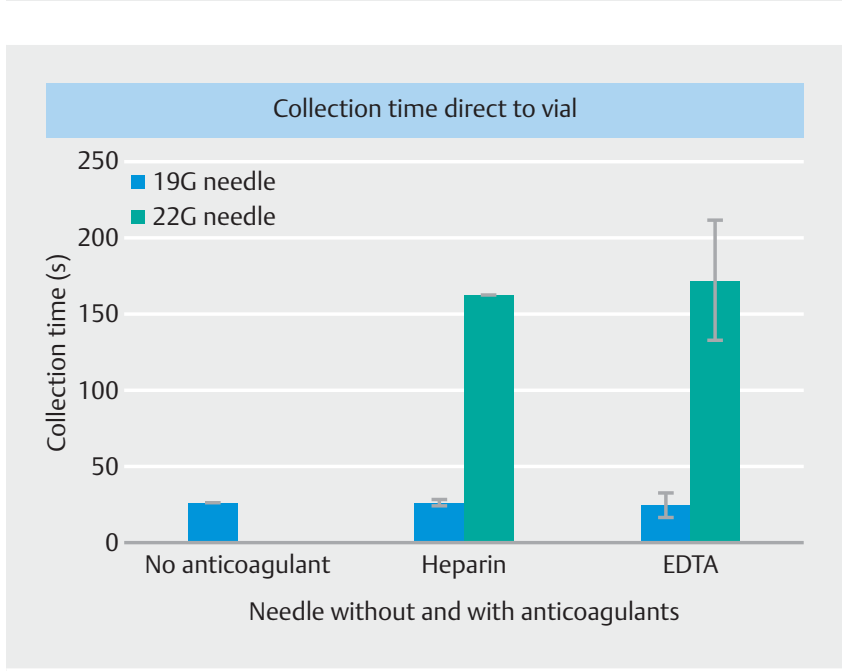

- Fig. 6 Mean small-volume $(10 \mathrm{~mL})$ blood collection times with direct-to-vial using 19G/22 G needles with/without anticoagulants. The $22 \mathrm{G}$ saline-coated needle did not finish due to clotting. The $19 \mathrm{G}$ heparin/EDTA-coated needles had similar mean blood collection times compared to saline-coated needles $(P>0.05)$.

When using the direct-to-vial method, clotting was a common problem with both $19 \mathrm{G}$ and $22 \mathrm{G}$ needles, which invariably led to incomplete sampling. For each vial of blood collection per trial, there was a tendency towards increased time for blood collection, when comparing the first vial to the fifth vial, regardless of needle gauge. Using the $22 \mathrm{G}$ needles, only three successfully completed blood collections were achieved, with an average time of 239 seconds. With the $19 \mathrm{G}$ needle, two incomplete blood collections occurred, both during trial two and after three prior successful blood collections with the same needle. Given the lack of successful complete blood collections, comparisons between $19 \mathrm{G}$ and $22 \mathrm{G}$ needles using direct-to-vial method and the suction syringe method could not be made. 
- Table 1 Mean small-volume blood collection times and standard deviations using direct-to-vial and suction syringe method for heparin/EDTAcoated 19G/22 G needles.

\begin{tabular}{|l|l|l|l|}
\hline Anticoagulant and needle gauge & Direct-to-vial method (sec) & Suction syringe method (sec) & P value \\
\hline Heparin-coated 19G needle $(n=2)$ & $25.5 \pm 1.7$ & $11.05 \pm 1.05$ & 0.059 \\
\hline EDTA-coated 19G needle $(n=2)$ & $24.0 \pm 5.4$ & $10.25 \pm 0.25$ & 0.111 \\
\hline Heparin-coated 22 G needle $(n=2)$ & $162 \pm 1$ & $136.5 \pm 4.5$ & 0.085 \\
\hline EDTA-coated 22 G needle $(n=2)$ & $171.5 \pm 28.5$ & $142.5 \pm 2.5$ & 0.109 \\
\hline EDTA, ethylenediaminetetraacetic acid. & & & \\
\hline
\end{tabular}

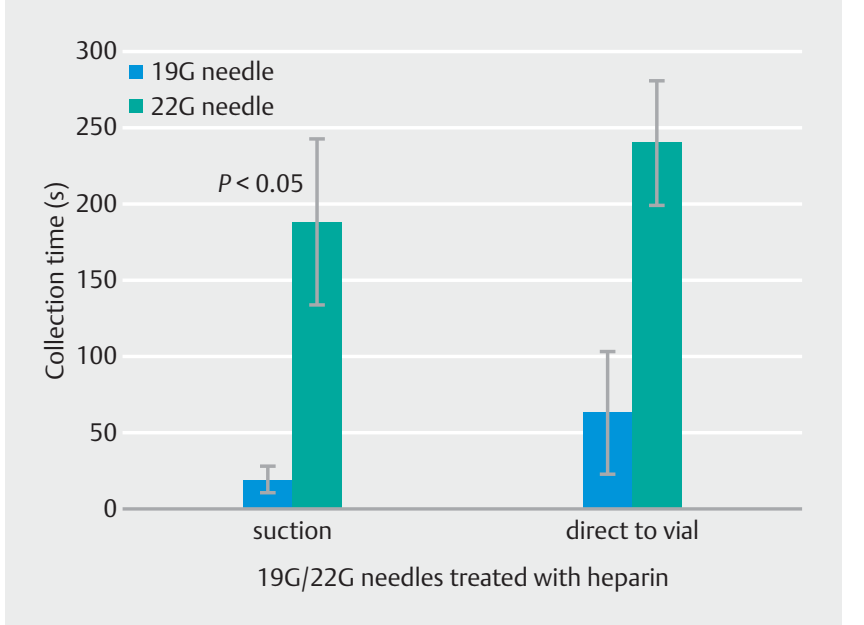

- Fig. 7 Mean large-volume ( $25 \mathrm{~mL}$ ) blood collection times in 19G/ $22 \mathrm{G}$ heparin-coated needles stratified by suction syringe and direct-to-vial methods. When using the direct-to-vial method, clotting was a common problem with both $19 \mathrm{G}$ and $22 \mathrm{G}$ needles. Only three successful blood collections were completed with $22 \mathrm{G}$ needles.

Complications that were encountered included incomplete blood collections when using the direct-to-vial method as described above. There was significant bleeding in two of 15 (13\%) blood collections when using the $19 \mathrm{G}$ needles that resulted in slight oozing at the puncture site after 3 minutes and resolved with manual pressure. No bleeding complications were observed when using the $22 \mathrm{G}$ needles.

\section{Blood cell enumeration}

We evaluated the complete blood counts of the blood collected. As shown in $>$ Table 2 , no significant differences in the white blood cells, red blood cells, and platelets were detected in the portal blood collected using either 19G or $22 \mathrm{G}$ needles. No shearing of any erythrocytes was mentioned in any of the samples.

\section{Bleeding trials}

The role of needle diameter in bleeding was determined. All puncture sites for both $19 \mathrm{G}$ and $22 \mathrm{G}$ needles had immediate bleeding. However, by 3 minutes, none of the puncture sites from the $22 \mathrm{G}$ needle showed any sign of bleeding, while two of $20(10 \%)$ of the puncture sites from the $19 \mathrm{G}$ needle had ongoing bleeding that required manual compression for hemostasis ( $\triangleright$ Table 3 ).

\section{Discussion}

To our knowledge, this is the first feasibility and safety study evaluating the use of a 22G EUS-FNA needle compared to a 19 G EUS-FNA needle for transhepatic portal vein sampling.

In 2015, Catenacci et. al first described the technique of EUS-guided portal vein sampling using a $19 \mathrm{G}$ needle [5]. The authors successfully showed EUS-guided portal vein sampling in 18 patients without any bleeding complications; however, the potential risk of bleeding remains a major concern when using a relatively large needle to sample the portal system. Subsequently, two additional studies have evaluated EUS-guided portal vein sampling with smaller gauge needles. Liu et. al successfully showed EUS-guided portal vein sampling in 29 patients using $20 \mathrm{G}$ needles without any bleeding complications [17]. Levy et. al then used $22 \mathrm{G}$ needles in EUS-guided portal vein sampling in 10 patients [18]. Thus, combining the three studies in the current literature, a total of 57 patients have been evaluated for EUS-guided portal vein sampling without any evidence of bleeding complications.

Further evaluation of potential bleeding complications with EUS and portal vein sampling can be drawn from studies evaluating EUS-guided portal vein access for measuring portal pressures and angiography. In several studies evaluating the use of EUS to measure portal pressures, 19 G, 22 G, and 25 G needles were used, but these studies did not involve blood collection or characterization of cellular integrity $[3,4,20,22]$. Notably, $19 \mathrm{G}$ needles were reported to cause intra-abdominal bleeding when evaluated for EUS-guided angiography in a swine model [22]. In a separate study, Tien et. al used a $21 \mathrm{G}$ needle for intra-operative portal vein sampling via direct puncture, at the time of surgery [15]. The authors reported bleeding associated with needle puncture, which were stopped in most cases by digital compression, except for one that required suture [15]. Given the potential serious complications of peritoneal hemorrhage and lack of any endoscopic treatments if one were to occur during the procedure, it would be sensible to use the smallest gauge needle possible. In the current study, we report the feasibility and safety of using a 22 G EUS needle for portal vein blood sampling with a pig model. 
- Table 2 Complete blood counts for samples collected with $19 \mathrm{G}$ and $22 \mathrm{G}$ needles.

\begin{tabular}{|l|l|l|l|}
\hline $\begin{array}{l}\text { Needle } \\
\text { gauge }\end{array}$ & $\begin{array}{l}\text { White blood cells } \\
(\mathbf{K} / \boldsymbol{\mu L})\end{array}$ & $\begin{array}{l}\text { Red blood cells } \\
\mathbf{( M / \mu L )}\end{array}$ & $\begin{array}{l}\text { Platelets } \\
\mathbf{( K / \mu L )}\end{array}$ \\
\hline $\begin{array}{l}19 \mathrm{G} \\
(\mathrm{n}=4)\end{array}$ & $11.76 \pm 1.46$ & $5.89 \pm 0.65$ & $546 \pm 112$ \\
\hline $22 \mathrm{G}$ & $11.47 \pm 1.00$ & $5.91 \pm 0.13$ & $541 \pm 69$ \\
\hline$(n=4)$ & & \\
\hline
\end{tabular}

- Table 3 Bleeding trials with saline-coated $19 \mathrm{G}$ and $22 \mathrm{G}$ needles showing all puncture sites with both needles had immediate bleeding.

\begin{tabular}{|l|l|l|}
\hline Needle gauge & $\begin{array}{l}\text { Immediate } \\
\text { bleeding }\end{array}$ & $\begin{array}{l}\text { Delayed } \\
\text { bleeding }\end{array}$ \\
\hline Saline-coated 19 G needle $(n=20)$ & $20(100)$ & $2(10)$ \\
\hline Saline-coated 22 G needle $(n=20)$ & $20(100)$ & $0(0)$ \\
\hline
\end{tabular}

In this study, we did not find any bleeding complications with $22 \mathrm{G}$ needles, while there was a $10 \%$ and $15 \%$ bleeding complication rate with $19 \mathrm{G}$ needles in our bleeding trial and portal vein sampling trial, respectively. While this finding may be difficult to apply to human patients, any potential reduction in bleeding risk is important, especially if portal venous sampling were to become a routine diagnostic test as part of a cancer staging process. It should be noted that the approach to access to the portal vein, namely via the transhepatic or extrahepatic routes, may impact on safety. In our study, we only performed intrahepatic portal vein sampling as there is a potential benefit of the liver parenchyma tamponading the needle track and limiting bleeding complications [4, 15]. Understandably, obtaining blood from the larger, main extrahepatic portal vein would be easier endosonographically. Given the bleeding complications shown in our study with $19 \mathrm{G}$ needles using the transhepatic approach, however, we would expect even higher rates of bleeding using an extrahepatic approach given the absence of the liver's tamponading effect. The benefit of the liver's tamponading effect is highlighted with the study by Tien et. al, which evaluated direct puncture of the portal vein with $21 \mathrm{C}$ needles. All patients required digital compression to stop bleeding, and one patient further required suturing to stop the bleeding after needle puncture [15].

The main strength of this study was evaluating the effect of priming the EUS-FNA needles with anticoagulants. EUS-FNA needles are not designed for blood acquisition and may be prone to clotting. After access to the portal vein is obtained and negative suction is applied, blood from the portal vein has to travel the length of the echoendoscope. While larger-gauge needles have not been shown to have clotting issues [5, 17], 22 G needles were found to have "slow" aspiration, which was corrected by adding heparin to the collection tubes [18]. Similarly, our results showed that the saline-coated $22 \mathrm{G}$ needle was incapable of obtaining an adequate quantity of portal vein blood to run a
CTC enumeration test due to clotting within the needle. Furthermore, needles coated with either heparin or EDTA facilitated portal venous blood collection; however, there was a tendency towards proportionally longer blood collection time when multiple vials of blood were being drawn through the same FNA needle, independent of its caliber. For instance, in our third trial with the $19 \mathrm{G}$ needle, the first vial had a blood collection time of $12.1 \mathrm{~s}$, while the fifth vial required $40.3 \mathrm{~s}$. There are two possible explanations for this finding. First, the amount of heparin in the needle is likely decreased with each additional blood collection leading to more clotting and hence an increase in blood collection time. Furthermore, the increased time needed for blood collection likely allows further activation of the extrinsic coagulation cascade both around and within the needle shaft and facilitate clotting. Despite this limitation, even the smaller caliber $22 \mathrm{G}$ heparin-coated EUS needle was able to consistently obtain a large volume of portal venous blood $(25 \mathrm{~mL})$ using the suction syringe method, which should be more than adequate for CTC analysis. A special note should be made about the amount of heparin that is added given its potential implications on blood testing. Levy et. al added $2 \mathrm{~mL}(10,000 \mathrm{U})$ of heparin to Streck tubes, and subsequent molecular analysis testing could not be completed in any of the FIVE blood samples with heparin [18]. In our study, only $100 \mathrm{uL}(100 \mathrm{U})$ was added, which was $1 \%$ the amount used in Levy et. al's study. Molecular analysis testing was beyond the scope of this study, but potentially the amount of heparin used, and the type of anticoagulant used can affect subsequent testing. Future studies will need to be performed for definitive evaluation.

Regarding our testing with EDTA-coated needles, safety may be an issue as this is not a common agent used in patients, especially when compared to heparin. However, EDTA is approved by the FDA for use in treating lead poisoning and toxicity form other heavy metals as an infusion, up to $1 \mathrm{~g} /$ day [23]. In our study, there was approximately at most 100 uL of EDTA in the needle, which is $1.8 \mathrm{ug}$ of EDTA. In addition, since negative pressure is being constantly applied through the needle for suctioning, realistically the amount potentially entering the patient is inconsequential.

In addition to showing the feasibility and safety of collecting portal blood, we also showed that blood cell enumeration was successful with smaller gauge needles. There is the potential for lysis of cells through shear forces as FNA needles are not designed for blood acquisition [24]. This may be especially true for smaller gauge needles given Poiseuille's Law, and pressure is inversely equal to the radius to the fourth power [25]. The results of our study suggest that blood cell enumeration was not an issue for smaller FNA needles.

Another strength of our study was evaluating different blood collection methods. Previous studies have only evaluated the suction syringe method. We wanted to test the suction syringe method and direct-to-vial collection method to identify the optimal choice of portal vein blood collection. We hypothesized that the direct-to-vial collection method may be preferred by removing the need to transfer the blood sample, which is needed in the suction syringe collection method. However, for large volume sampling, we showed that the direct-to-vial collection 
method had clotting issues that led to incomplete blood collections, regardless of the needle gauge. While the direct-to-vial method is more convenient as it would eliminate the need to transfer the sample from a suction syringe to a sample vial, the latter collection method is preferred because it can avoid clotting in large volume sampling. The direct-to-vial method can potentially be used for small-volume collections as long as the $22 \mathrm{G}$ needle is coated with heparin. Additionally, we did not account for the transfer time during the suction syringe method. We anticipate needing 30 to 40 seconds to transfer a $5-\mathrm{mL}$ blood sample from the suction syringe to a vacutainer. Therefore, blood collection times may be significantly shorter with the direct-to-vial method, and this method may be preferred for small-volume collections.

Our results provide insights into further refining the process of EUS-guided portal vein blood sampling, which has emerged as an important minimally invasive approach to collect and analyze CTCs. Current 5-year mortality for patients with PDAC with R0 resection is $20 \%$ to $30 \%$ [26], likely as a result of micrometastasis from shedding of CTCs from the primary tumor before clinical or radiologic evidence of metastases. Circulating tumor cells are rarely found in peripheral blood in part due to the filtering effects of the liver and the capillaries, especially in the pre-metastatic stage $[5,14]$, and CTC numbers are much higher in the portal venous blood and can be more readily detected $[15,16]$. Previous studies of CTCs in the portal blood collected intraoperatively in patients with resectable PDAC showed that patients with CTCs in the portal vein had a higher rate of liver metastases compared to patients without detectable CTCs $[15,27]$. The prognostic and treatment values of CTCs may be expanded with more ready and safe sampling of portal vein blood.

There are several limitations in our study. First, this is a feasibility and safety study of portal vein sampling using a swine model, and further studies in human patients are needed to confirm our findings. Nevertheless, previous studies using swine models showed a similar baseline portal vein pressures of 5 to $10 \mathrm{mmHg}$ comparable to that of humans [17, 22]. Additionally, swine models have been successfully used to simulate hepatic bleeding with increased rates of bleeding with larger gauge needles compared to smaller gauge needles especially with anticoagulation $[23,24]$. Therefore, similar results should be expected in human patients. Second, our procedures involved an intraoperative ultrasound-guided transhepatic portal vein access rather than the endoscopic approach. We predict that these differences will not be significant given that we used the same EUS-FNA needle that we would have used in an endoscopic approach as well as artificially creating a U-loop in the needle to mimic the intragastric loop expected to form in the stomach during an endoscopy. Identifying an intrahepatic portal vein would not be too difficult as this is routinely examined during our EUS exams. It maybe, however, challenging to try to maintain a stable position for up to 136 seconds to collect portal blood in human patients. Certain patients such as those with cirrhosis, may make it difficult to identify and access the intrahepatic portal vein given liver parenchymal changes and harder caliber of the portal vein as well.

\section{Conclusion}

In summary, we have demonstrated in an animal model that it is feasible and safe to use $22 \mathrm{G}$ needles for portal vein blood sampling. In particular, needles coated with a small quantity of an anticoagulant, such as low volume heparin, EDTA or citrate, can prevent or reduce clotting in the process and improve efficiency of blood sampling. Syringe suctioning is preferred to the direct-to-vial method for blood collection, especially if collecting large volumes, as it reduces the chances of needle clogging and blood clotting. These results can form the basis for further studies of EUS-guided portal vein sampling in humans. The establishment of a safe and efficient method for portal vein blood analysis has important implications in the management of patients with pancreaticobiliary cancers.

\section{Competing interests}

The authors declare that they have no conflict of interest.

\section{References}

[1] Siegel RL, Miller KD, Jemal A. Cancer statistics, 2019. CA Cancer J Clin 2019; 69: 7-34

[2] Jani BS, Rzouq F, Saligram S et al. Endoscopic ultrasound-guided fineneedle aspiration of pancreatic lesions: a systematic review of technical and procedural variables. N Am J Med Sci 2016; 8: 1-11

[3] Huang JY, Samarasena JB, Tsujino T et al. EUS-guided portal pressure gradient measurement with a simple novel device: a human pilot study. Gastrointest Endosc 2017; 85: 996-1001

[4] Lai L, Poneros J, Santilli j et al. EUS-guided portal vein catheterization and pressure measurement in an animal model: a pilot study of feasibility. Gastrointest Endosc 2004; 59: 280-283

[5] Catenacci DV, Chapman CG, Peng X et al. Acquisition of portal venous circulating tumor cells from patients with pancreaticobiliary cancers by endoscopic ultrasound. Gastroenterology 2015; 149: 1794-1803 e4

[6] Lin E, Cao T, Nagrath S et al. Circulating tumor cells: diagnostic and therapeutic applications. Annu Rev Biomed Eng 2018; 20: 329-352

[7] Massague J, Obenauf AC. Metastatic colonization by circulating tumour cells. Nature 2016; 529: 298-306

[8] Cristofanilli M, Budd GT, Ellis M] et al. Circulating tumor cells, disease progression, and survival in metastatic breast cancer. N Engl J Med 2004; 351: 781-791

[9] Cohen S], Punt CJA, lannotti N et al. Relationship of circulating tumor cells to tumor response, progression-free survival, and overall survival in patients with metastatic colorectal cancer. J Clin Oncol 2008; 26: 3213-3221

[10] Pachmann K, Camara O, Kavallaris A et al. Monitoring the response of circulating epithelial tumor cells to adjuvant chemotherapy in breast cancer allows detection of patients at risk of early relapse. J Clin Oncol 2008; 26: 1208-15

[11] Hao SJ, Wan Y, Xia Y et al. Size-based separation methods of circulating tumor cells. Adv Drug Deliv Rev 2018; 125: 3-20

[12] Heitzer E, Haque IS, Roberts CES et al. Current and future perspectives of liquid biopsies in genomics-driven oncology. Nat Rev Genet 2019; 20: 71-88 
[13] Rossi G, Ignatiadis M. Promises and pitfalls of using liquid biopsy for precision medicine. Cancer Res 2019; 79: 2798-2804

[14] Kurihara T, Itoi T, Sofuni A et al. Detection of circulating tumor cells in patients with pancreatic cancer: a preliminary result. J Hepatobiliary Pancreat Surg 2008; 15: 189-195

[15] Tien YW, Kuo H, Ho B et al. A high circulating tumor cell count in portal vein predicts liver metastasis from periampullary or pancreatic cancer: a high portal venous CTC count predicts liver metastases. Medicine (Baltimore) 2016; 95: e3407

[16] Rahbari NN, Bork U, Kircher A et al. Compartmental differences of circulating tumor cells in colorectal cancer. Ann Surg Oncol 2012; 19 : 2195-2202

[17] Liu X, Li C, Li J et al. Detection of CTCs in portal vein was associated with intrahepatic metastases and prognosis in patients with advanced pancreatic cancer. J Cancer 2018; 9: 2038-2045

[18] Levy MJ, Kipp BR, Milosevic D et al. Analysis of cell-free DNA to assess risk of tumoremia following endoscopic ultrasound fine-needle aspiration of pancreatic adenocarcinomas. Clin Gastroenterol Hepatol 2018; 16: 1632-1640 e1

[19] Chapman CG, Waxman I. EUS-guided portal venous sampling of circulating tumor cells. Curr Gastroenterol Rep 2019; 21: 68

[20] Schulman AR, Thompson CC, Ryou M. EUS-guided portal pressure measurement using a digital pressure wire with real-time remote dis- play: a novel, minimally invasive technique for direct measurement in an animal model. Gastrointest Endosc 2016; 83: 817-20

[21] Wang H, Hara Y, Liu X et al. Detection and enumeration of circulating tumor cells based on their invasive property. Oncotarget 2015; 6: 27304-27311

[22] Magno P, Ko C, Buscaglia JM et al. EUS-guided angiography: a novel approach to diagnostic and therapeutic interventions in the vascular system. Gastrointest Endosc 2007; 66: 587-591

[23] Lamas GA, Boineau R, Goertz C et al. EDTA chelation therapy alone and in combination with oral high-dose multivitamins and minerals for coronary disease: The factorial group results of the Trial to Assess Chelation Therapy. Am Heart J 2014; 168: 37-44.e5

[24] Bowen RA, Remaley AT. Interferences from blood collection tube components on clinical chemistry assays. Biochem Med (Zagreb) 2014; $24: 31-44$

[25] Pfitzner J. Poiseuille and his law. Anaesthesia 1976; 31: 273-5

[26] Neoptolemos JP, Stocken DD, Friess $\mathrm{H}$ et al. A randomized trial of chemoradiotherapy and chemotherapy after resection of pancreatic cancer. N Engl J Med 2004; 350: 1200-1210

[27] Bissolati M, Sandri MT, Burtulo G et al. Portal vein-circulating tumor cells predict liver metastases in patients with resectable pancreatic cancer. Tumour Biol 2015; 36: 991-996 